\title{
Retrospective analysis of feline intestinal parasites: trends in testing positivity by age, USA geographical region and reason for veterinary visit
}

\author{
Sarah Sweet, Donald Szlosek, Donald McCrann, Michael Coyne* (i), David Kincaid and Evan Hegarty
}

\begin{abstract}
Background: The goals of this retrospective study were to estimate parasite positivity in samples from cats using zinc sulfate fecal flotation by centrifugation ("centrifugation") and coproantigen and examine trends with age, geographical region and reason for visit to veterinarian. Common methods of parasite detection, such as centrifugal flotation, passive flotation, or direct smear, may underrepresent the true prevalence of intestinal parasites in cats. Coproantigen testing detects more positive samples than traditional methods alone.

Methods: Feline fecal test results from the continental USA containing results for fecal exams performed using centrifugation paired with coproantigen results for ascarid, hookworm, whipworm and Giardia were obtained from the database of a national commercial reference laboratory comprised of multiple regional sites.

Results: Parasite positivity was highest in samples from young cats and decreased with cat age. The western region of the USA had lower total parasite positivity than other regions for all parasites except Giardia. Cats receiving fecal tests during veterinary wellness visits had only slightly lower parasite positivity than samples from cats during sick clinical visits.

Conclusions: This study showed a larger population of cats are at increased risk of parasitism than commonly believed and coproantigen testing produces more positive test results for the four parasites that antigen can detect than centrifugation of feline fecal samples.
\end{abstract}

Keywords: Cat, Feline, Parasitism, Fecal, Flotation, Coproantigen, Hookworm, Whipworm, Ascarid, Giardia

\section{Background}

Intestinal parasite risk to pet cats is underrepresented in the literature and is often overlooked by pet owners and veterinarians [1-3]. The aims of this study were to report the proportion of positive fecal test results in samples from pet cats in the United States; to examine trends in positivity with age, geographical region, and reason for veterinary visit; and to describe differences in positivity between the two diagnostic methods, centrifugation and

${ }^{*}$ Correspondence: Michael-Coyne@idexx.com

IDEXX Laboratories, Inc., One IDEXX Drive, Westbrook, ME, USA coproantigen immunoassay. Previously published studies investigating feline intestinal parasitism in the USA were limited to a single parasite $[1,4,5]$, a particular geographical region $[2,3,6]$, or focused solely on free-roaming or shelter cats $[2,3,7]$. These limitations make it difficult for a veterinarian to assess the risk to their patients.

Reported prevalence of intestinal parasite infection in domestic cats is often region-specific and may vary widely, ranging from 0.03 to $33 \%$ [8-12]. One resource for national reporting on feline fecal parasitism is CAPC (Companion Animal Parasite Council) [13] which sources its data from fecal submissions to commercial 
veterinary reference laboratories (IDEXX Reference Laboratories, Inc., Westbrook, ME, USA, Antech Diagnostics and Imaging, Fountain Valley, CA USA). While CAPC prevalence maps provide local, state and regional data, they ignore age and health status of the patient.

Fecal flotation is the most commonly used method of parasite identification in veterinary medicine. This technique may underrepresent prevalence due to prepatent or single-sex infections [14] and when conducted in clinic, result validity is highly dependent on sample preparation, type and concentration of flotation solution and reader skill $[15,16]$. Coproantigen tests have previously been shown to increase the ability to detect infections [12]. The addition of coproantigen immunoassay detection to fecal diagnostics should increase the detection of feline intestinal parasite infections in the USA.

\section{Methods}

Results ordered by veterinary practices in the USA were collected from samples submitted to a national commercial reference laboratory corporation using proprietary software (IDEXX Laboratories, Inc. Westbrook, ME, USA) from January 2017 to December 2018. A single centrifugation for $5 \mathrm{~min}$ at $500 \times g$ was performed using zinc sulfate flotation solution (specific gravity 1.24 ) then read at $100 \times$ and $400 \times$ magnifications following standard methods [17]. Coproantigen immunoassays utilized unique capture and detection antibodies developed against recombinantly expressed proteins of ascarids (Toxocara spp., Toxascaris spp., Baylisascaris spp.), hookworms (Ancylostoma spp., Uncinaria spp.) and whipworms (Trichuris spp.) and Giardia spp. [17-19]. Presence of Cystoisospora spp., lungworms (Filaroides spp., Aelurostrongylus spp., Angiostrongylus spp., Eucoleus spp., Capillaria spp.), tapeworms (cestodes) (Dipylidium spp., Moniezia spp., Anoplocephala spp., Spirometra spp., Taenia spp., Mesocestoides spp., Diphyllobothrium spp., Echinococcus spp., Hymenolepis spp., Paranoplocephala spp., Cittotaenia spp.) and trematodes (Paragonimus spp., Alaria spp., Nanophyetus spp., Platynosomum spp.), was only detected via centrifugation and these parasites are reported as well.

Samples from cats two months to 25 years of age, tested for hookworm, ascarid, whipworm and Giardia spp. with centrifugation and coproantigen (IDEXX Laboratories, Inc. Westbrook, ME, USA) on the same visit were used in the study. To examine if there was bias in submission, e.g. samples suspected of being positive were more likely to be submitted for both tests, centrifugation positivity by parasite for these groups was compared. While centrifugation positive percentages tended to be higher in samples submitted for testing by both methods, the differences did not exceed 0.5 percentage points for any parasite.
For inclusion in the study, age and geographical location of the clinic that provided the sample were required. For cats with multiple samples within the dataset, only the first sample for an individual cat was included in the analysis. Only samples from clinical visits were included; samples from visits that were classified as retail, grooming, or boarding were excluded. Clinical visits were subdivided into wellness and other clinical visits. Wellness visits were visits to the veterinarian for annual exams, vaccinations, or routine check-ups. Other clinical visits were those for a new illness, monitoring an existing illness, or for a procedure such as surgery or teeth cleaning.

All data analyses were conducted using $\mathrm{R}$ version 3.5.3 [20]. Data analysis was done with the tidyverse and multiple helper functions within the following packages: data. table [21], magrittr [22], here [23], Hmisc [24], ggplot2 [25], gridExtra [26], ggpubr [27] and extrafont [28]. Summary statistics were reported as the percent positive of all test results. Confidence intervals were calculated using the binomial exact method. Significant differences in percent positive among subgroups of samples were determined by non-overlapping confidence intervals. For regional analysis of parasite positivity, United States Census Bureau regions were used, as these align with major geographical and demographical differences that could theoretically influence parasite prevalence [29].

\section{Results}

A total of 1,271,460 feline fecal test results were obtained. Fecal test results from cats younger than two months and older than 25 years of age $(n=109,046)$ and any subsequent test results on cats already in the study $(n=$ $197,557)$ were excluded. Sample results obtained from cats during non-clinical visits $(n=561,681)$, with missing geographical data $(n=4202)$, or not tested with both coproantigen and centrifugation $(n=303,171)$ were also excluded. The study population was comprised of fecal test results from 95,803 cats, with 51.0\% ( $n=48,801)$ obtained during wellness visits and 49.0\% $(n=47,002)$ obtained during other clinical visits (Fig. 1). Approximately, $1.4 \%$ of the test results had more than one parasite identified. The highest co-infection was ascarid + Giardia which occurred in $0.9 \%$ of all fecal tests. Additional co-infection information can be found in Additional file 1: Table S1.

In the study population, ascarids were the most common parasites identified by centrifugation, with a positivity of $4.6 \%$, followed by Cystoisospora (2\%), Giardia (1.7\%), tapeworms (0.9\%), hookworms (0.5\%), lungworms $(0.3 \%)$ and whipworms (0.03\%) (Fig. 2, Table 1$)$. Ascarids and Giardia had the highest percent positive on coproantigen with $6.2 \%$ and $6.5 \%$ positive results, respectively. For each of the intestinal parasites tested by both 


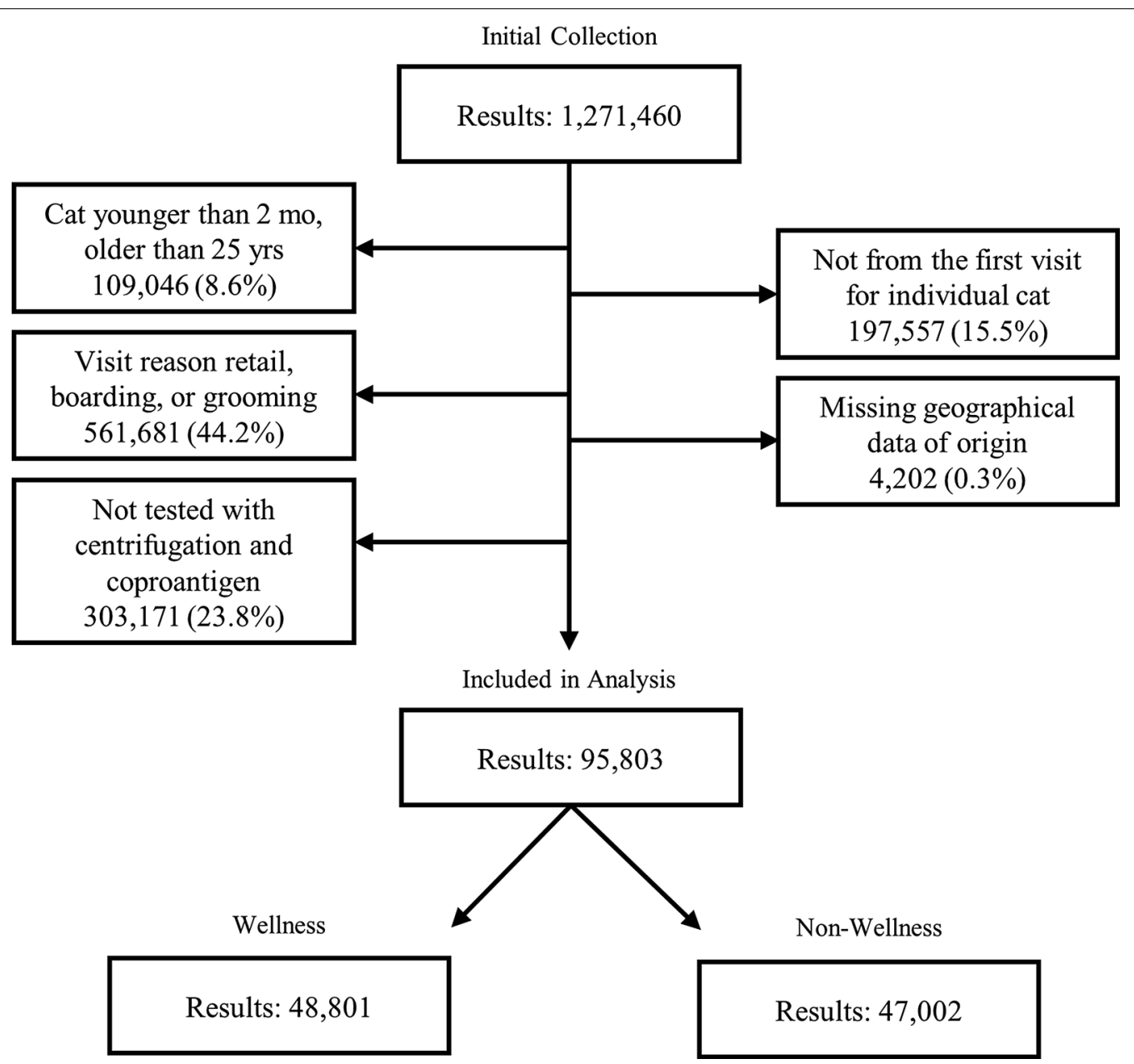

Fig. 1 Consort diagram of the exclusion criteria and study population

diagnostic methods, the proportion of positive tests was significantly higher by coproantigen than by centrifugation (Table 1).

Most fecal samples that were positive for a parasite by centrifugation also tested positive by coproantigen for the same parasite, excepting whipworms, which had slightly less than half of the positives by centrifugation also positive by coproantigen. Of the fecal samples that tested positive for ascarids by either method, $7.7 \%$ were positive by centrifugation alone, $32.0 \%$ were positive by coproantigen alone and the remaining $60.3 \%$ were positive by both methods. For Giardia, the positivity by centrifugation alone was $1 \%, 74.7 \%$ by antigen alone and $24.3 \%$ by both. For hookworms, the positivity by centrifugation alone was $17.4 \%, 56.8 \%$ by antigen alone and $25.8 \%$ by both. For whipworms, the positivity by centrifugation alone was $10.4 \%, 80.4 \%$ by antigen alone and $9.2 \%$ by both. Numbers of positive tests by each method are detailed in Additional file 1: Table S1.
A general trend of decreasing positive proportions of test results was observed as the age of the cat increases (Fig. 3). However, examining the age trend in positivity by parasite showed that for Giardia, hookworms, tapeworms and whipworms, the highest proportion of positive results was on samples from cats either 7-12 months, or 1-2 years of age (Fig. 4, Table 1). These trends are consistent between centrifugation and coproantigen methods, where applicable. The ages of cats receiving centrifugation or coproantigen tests on wellness or non-wellness veterinary visits can be found in Additional file 2: Table S2.

Combined parasite test result positivity varied by region (including ascarids, Giardia, hookworms and whipworms). The lowest overall parasite positivity was found in samples from the western region, though Giardia positivity in this region was no less frequent than in other regions. The highest proportions of ascarid-positive samples were from the Northeast and the Midwest 


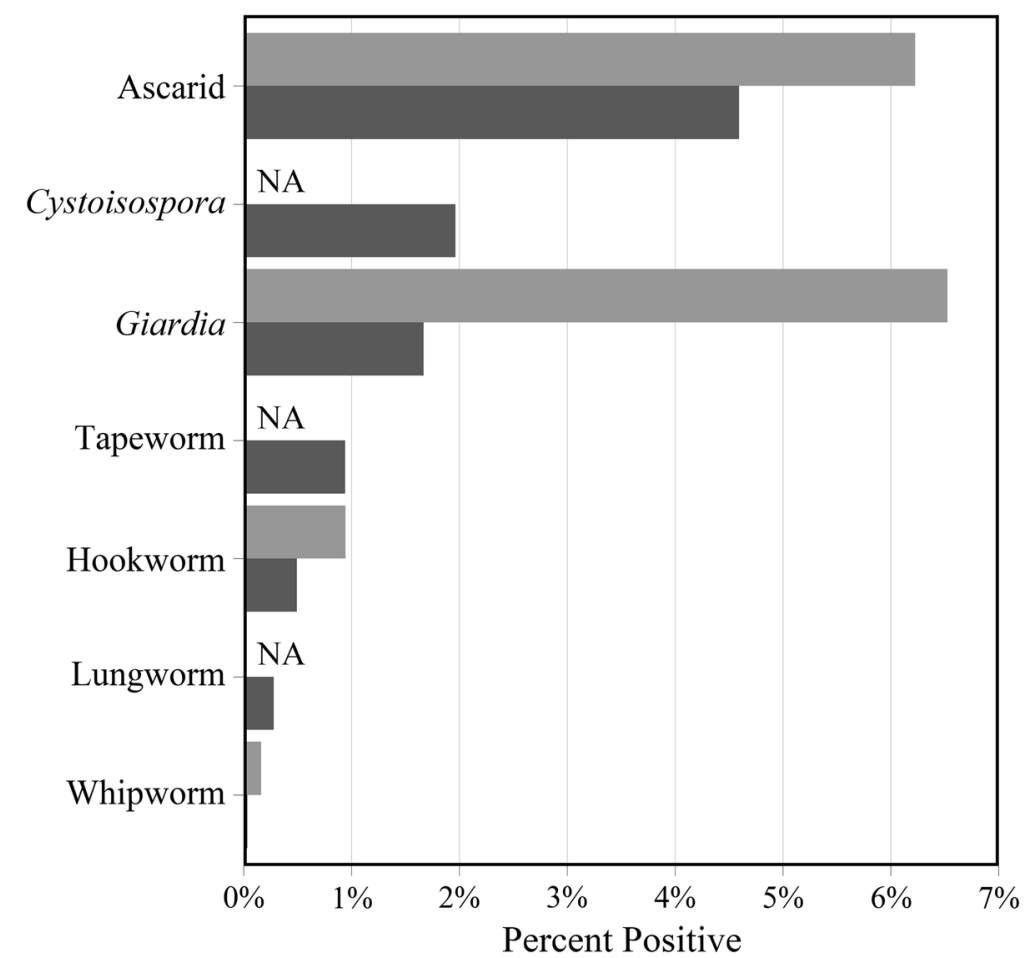

Method

Centrifugation

Coproantigen

Percent Positive

Fig. 2 Proportion of cats with a positive test result by centrifugation or coproantigen

Table 1 Positive test results for feline intestinal parasites by centrifugation and coproantigen by age

\begin{tabular}{|c|c|c|c|c|c|c|c|c|c|}
\hline Parasite & Method & $\begin{array}{l}2-6 \text { months } \\
(\%) \\
(95 \% \mathrm{Cl})\end{array}$ & $\begin{array}{l}7-12 \text { months } \\
(\%) \\
(95 \% \mathrm{Cl})\end{array}$ & $\begin{array}{l}1-2 \text { years (\%) } \\
(95 \% \mathrm{Cl})\end{array}$ & $\begin{array}{l}3-5 \text { years }(\%) \\
(95 \% \mathrm{Cl})\end{array}$ & $\begin{array}{l}6-8 \text { years }(\%) \\
(95 \% \mathrm{Cl})\end{array}$ & $\begin{array}{l}9-13 \text { years }(\%) \\
(95 \% \mathrm{Cl})\end{array}$ & $\begin{array}{l}14+\text { years }(\%) \\
(95 \% \mathrm{Cl})\end{array}$ & $\begin{array}{l}\text { Total (\%) } \\
(95 \% \mathrm{Cl})\end{array}$ \\
\hline \multirow[t]{2}{*}{ Ascarid } & CE & $12.4(12.0-2.9)$ & $8.1(7.4-8.8)$ & $4.8(4.3-5.3)$ & $2.9(2.6-3.2)$ & $1.7(1.5-1.9)$ & $1.0(0.9-1.2)$ & $0.6(0.5-0.8)$ & $4.6(4.5-4.7)$ \\
\hline & $\mathrm{CO}$ & $\begin{array}{l}16.5(16.0- \\
17.0)\end{array}$ & $9.8(9.1-10.5)$ & $6.2(5.7-6.8)$ & $4.2(3.9-4.5)$ & $2.5(2.2-2.8)$ & $1.6(1.5-1.8)$ & $1.2(1.0-1.4)$ & $6.2(6.1-6.4)$ \\
\hline Cystoisospora & CE & $6.3(6.0-6.7)$ & & & $0.8(0.7-0.9)$ & $0.6(0.5-0.7)$ & $0.4(0.3-0.5)$ & $0.3(0.2-0.4)$ & $2.0(1.9-2.1)$ \\
\hline \multirow[t]{2}{*}{ Giardia } & CE & $3.2(3.0-3.5)$ & $4.8(4.4-5.4)$ & $2.6(2.3-3.0)$ & $1.5(1.3-1.7)$ & $0.8(0.6-0.9)$ & $0.2(0.2-0.3)$ & $0.2(0.1-0.3)$ & $1.7(1.6-1.8)$ \\
\hline & $\mathrm{CO}$ & $\begin{array}{l}12.4(11.9- \\
12.8)\end{array}$ & $\begin{array}{l}16.6(15.7- \\
17.5)\end{array}$ & $\begin{array}{l}11.0(10.4- \\
11.7)\end{array}$ & $6.1(5.7-6.5)$ & $2.8(2.5-3.1)$ & $1.4(1.3-1.6)$ & $1.0(0.9-1.2)$ & $6.5(6.4-6.7)$ \\
\hline Tapeworm & CE & $1.1(1.0-1.3)$ & $1.5(1.3-1.9)$ & $1.6(1.4-1.9)$ & $1.4(1.2-1.6)$ & $0.8(0.7-1.0)$ & $0.5(0.4-0.6)$ & $0.2(0.1-0.3)$ & $0.9(0.9-1.0)$ \\
\hline \multirow[t]{2}{*}{ Hookworm } & CE & $0.5(0.4-0.6)$ & $1.4(1.1-1.7)$ & $1.1(0.9-1.4)$ & $0.6(0.5-0.7)$ & $0.3(0.2-0.4)$ & $0.2(0.2-0.3)$ & $0.1(0.1-0.2)$ & $0.5(0.5-0.5)$ \\
\hline & $\mathrm{CO}$ & $1.2(1.1-1.4)$ & $1.8(1.5-2.2)$ & $1.8(1.6-2.1)$ & $1.0(0.8-1.2)$ & $0.6(0.5-0.8)$ & $0.4(0.4-0.5)$ & $0.4(0.3-0.6)$ & $0.9(0.9-1$. \\
\hline Lungworm & CE & $0.6(0.5-0.8)$ & $0.5(0.3-0.7)$ & $0.5(0.3-0.6)$ & $0.2(0.1-0.3)$ & $0.1(0.1-0.2)$ & $0.1(0.0-0.1)$ & $0.1(0.0-0.1)$ & $0.3(0.2-0$. \\
\hline \multirow[t]{2}{*}{ Whipworm } & CE & $0.1(0.0-0.1)$ & $0.1(0.0-0.2)$ & $0.1(0.0-0.2)$ & $0.0(0.0-0.1)$ & $0.0(0.0-0.0)$ & $0.0(0.0-0.0)$ & $0.0(0.0-0.0)$ & $0.0(0.0-0.0)$ \\
\hline & $\mathrm{CO}$ & $0.2(0.1-0.3)$ & $0.2(0.1-0.4)$ & $0.2(0.1-0.3)$ & $0.2(0.1-0.3)$ & $0.2(0.1-0.2)$ & $0.1(0.1-0.2)$ & $0.1(0.0-0.1)$ & $0.2(0.1-0$ \\
\hline
\end{tabular}

Abbreviations: $\mathrm{CE}$, centrifugation; $\mathrm{CO}$, coproantigen

(Table 2). Whipworm and hookworm infections were the highest in samples from the South. Both Cystoisospora and tapeworms were highest in samples from southern and western regions (Table 2). When examined by age, category and region, samples from younger cats tended to have higher rates of positivity for hookworms, ascarids and Giardia in every region with more positive results found by coproantigen (Fig. 5, Additional file 3: Figure $\mathrm{S} 1)$. In addition, this trend was also seen when centrifugation results from all parasites are combined (Fig. 6). 


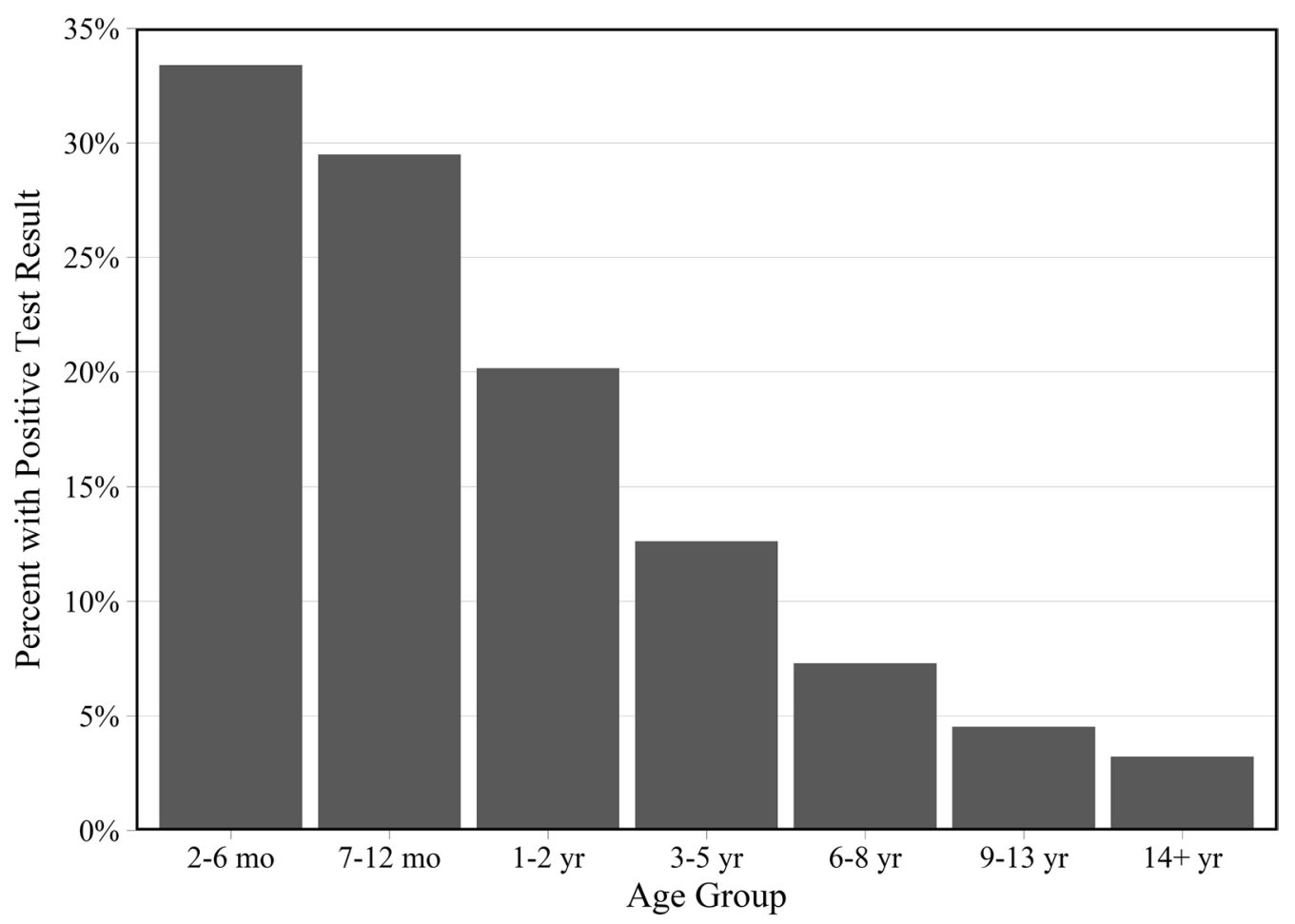

Fig. 3 Proportion of cats with a positive test result by either centrifugation or coproantigen by age

In sample results from cats presenting for a wellness exam, ascarids had the highest positivity by centrifugation (5.3\%), followed by Cystoisospora (2.2\%), Giardia (1.5\%), tapeworms $(0.8 \%)$, hookworms $(0.4 \%)$, lungworms $(0.2 \%)$ and whipworms $(<0.1 \%)$ (Table 3$)$. No trematodes were identified in any cat sample in this study. Similarly, in sample results from cats presenting for wellness exams, ascarids also had the highest positivity by coproantigen (7.2\%), followed by Giardia (6.2\%), hookworms (0.9\%) and whipworms (0.1\%) (Table 3). While parasite positivity was significantly different for most parasites on wellness compared to non-wellness clinical visits, the differences were not clinically meaningful. Ascarids and Cystoisospora were found more often on wellness exams. Age-group differences by clinical exam type were observed. Kittens 2-6-months of age comprised 28\% $(n=13,680)$ of the wellness exam population and only $14 \%(n=$ 6578 ) of the other clinical population (Additional file 4: Table S3). These age-group differences account for higher ascarid and Cystoisospora test positivity in wellness exams than other clinical visits, as kittens less than 6 months of age were disproportionately represented in wellness visits and have the highest rates of ascarid infections of any age group.

\section{Discussion}

The total parasite positivity rate in this study was largely driven by ascarid and Giardia test results regardless of method used. In addition, this study observed an agedependent relationship, with fewer positive test results seen in samples from adult cats as compared to those from cats under two years of age. Ascarid infections were highest in samples from 2-6-month-old kittens, likely owing to absence of early deworming protocols and vertical transmission from queen to offspring [30, 31].

Coproantigen testing detected more positive samples for hookworms, ascarids, whipworms and Giardia than centrifugation. Coproantigen testing for nematode parasites uses ELISA immunoassay technology to detect antigens secreted or excreted by the parasite of interest. These antigens are unique to the mature, metabolically active parasites and do not cross-react with eggs [1618]. Giardia spp. coproantigen testing detects a soluble cell wall antigen that is produced when the trophozoite encysts in the intestinal wall of the host [32]. Possible contributing factors to the difference in positive results may be difficulty identifying cysts or trophozoites via centrifugation, as with Giardia spp., or samples collected during a non-egg-shedding stage of the parasite's life-cycle. Combined positive results for hookworms, 


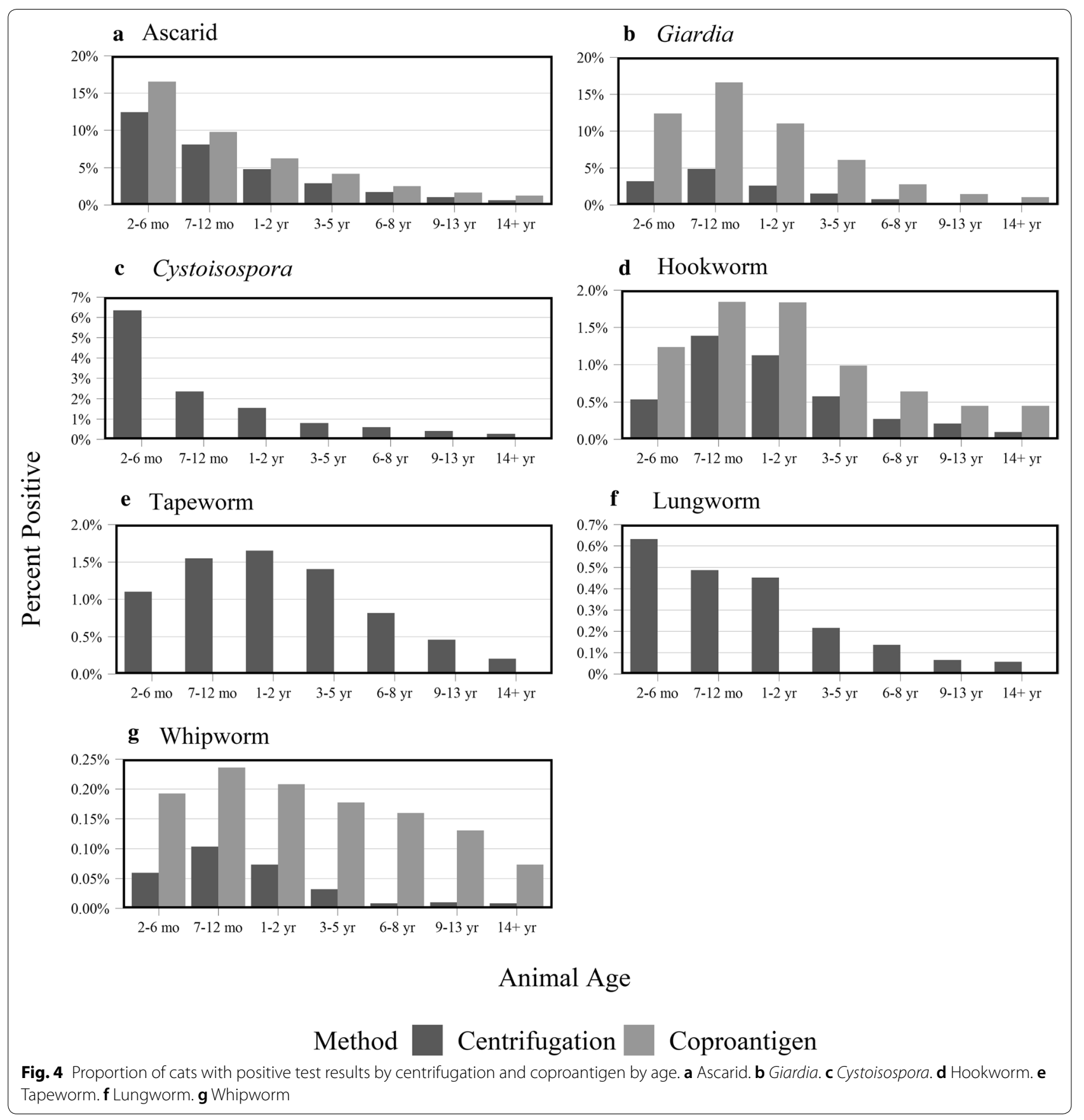

ascarids, whipworms and Giardia using coproantigen showed similar trends among samples from all age groups and regions with a consistently higher number of positive results than centrifugation.

The most notable regional difference in proportion of positive tests was in the western USA, which had a lower proportion of positives than all other regions detected using either centrifugation or coproantigen testing. Proportion of positive samples declined with age in each region, with possible deviations from this age-related positivity trend seen with whipworms. Samples from the West had a spike in overall parasite positivity in the 7-12month age range, inconsistent with trends in the other regions. This is because ascarid infections decreased in positivity with age of the cat and comprised most of the positive results in the South, the Northeast and the Midwest, whereas Giardia was the most common source of positive results in the West. The highest rate of Giardia 
Table 2 Proportion of positive test results across all parasites by United States Census Bureau regions

\begin{tabular}{|c|c|c|c|c|c|c|c|c|}
\hline \multirow[t]{2}{*}{ Parasite } & \multicolumn{2}{|l|}{ Midwest } & \multicolumn{2}{|l|}{ Northeast } & \multicolumn{2}{|l|}{ South } & \multicolumn{2}{|l|}{ West } \\
\hline & $\begin{array}{l}\text { Centrifugation } \\
(\%) \\
(95 \% \mathrm{Cl})\end{array}$ & $\begin{array}{l}\text { Coproantigen } \\
(\%) \\
(95 \% \mathrm{Cl})\end{array}$ & $\begin{array}{l}\text { Centrifugation } \\
(\%) \\
(95 \% \mathrm{Cl})\end{array}$ & $\begin{array}{l}\text { Coproantigen } \\
(\%) \\
(95 \% \mathrm{Cl})\end{array}$ & $\begin{array}{l}\text { Centrifugation } \\
(\%) \\
(95 \% \mathrm{Cl})\end{array}$ & $\begin{array}{l}\text { Coproantigen } \\
(\%) \\
(95 \% \mathrm{Cl})\end{array}$ & $\begin{array}{l}\text { Centrifugation } \\
(\%) \\
(95 \% \mathrm{Cl})\end{array}$ & $\begin{array}{l}\text { Coproantigen } \\
(\%) \\
(95 \% \mathrm{Cl})\end{array}$ \\
\hline Ascarid & $6.2(5.8-6.5)$ & $8.2(7.8-8.6)$ & $5.8(5.6-6.1)$ & $8.0(7.7-8.2)$ & $3.9(3.6-4.2)$ & $5.2(4.9-5.6)$ & $1.4(1.2-1.6)$ & $2.0(1.8-2.2)$ \\
\hline Cystoisospora & $1.8(1.6-2.0)$ & - & $1.8(1.7-1.9)$ & - & $2.2(1.9-2.4)$ & - & $2.2(2.0-2.5)$ & - \\
\hline Giardia & $2.0(1.8-2.2)$ & $7.5(7.1-7.9)$ & $1.4(1.3-1.5)$ & $6.6(6.4-6.9)$ & $1.7(1.5-1.8)$ & $5.9(5.5-6.2)$ & $2.0(1.8-2.2)$ & $6.1(5.8-6.4)$ \\
\hline Tapeworm & $0.7(0.6-0.8)$ & - & $0.7(0.6-0.8)$ & - & $1.1(1.0-1.3)$ & - & $1.5(1.3-1.7)$ & - \\
\hline Hookworm & $0.5(0.4-0.6)$ & $1.0(0.8-1.1)$ & $0.4(0.4-0.5)$ & $0.9(0.8-1.0)$ & $1.1(0.9-1.3)$ & $1.7(1.5-1.9)$ & $0.1(<0.1-0.1)$ & $0.4(0.3-0.5)$ \\
\hline Lungworm & $0.3(0.3-0.4)$ & - & $0.4(0.4-0.5)$ & - & $0.1(0.1-0.2)$ & - & $\begin{aligned}<0.1(< \\
0.1-0.1)\end{aligned}$ & - \\
\hline Whipworm & $<0.1(<0.1-0.1)$ & $0.2(0.1-0.2)$ & $\begin{array}{l}<0.1(<0.1-< \\
\quad 0.1)\end{array}$ & $0.1(0.1-0.2)$ & $0.1(0.1-0.2)$ & $0.3(0.2-0.4)$ & $\begin{array}{l}<0.1(<0.1-< \\
\quad 0.1)\end{array}$ & $0.1(0.1-0.2)$ \\
\hline
\end{tabular}

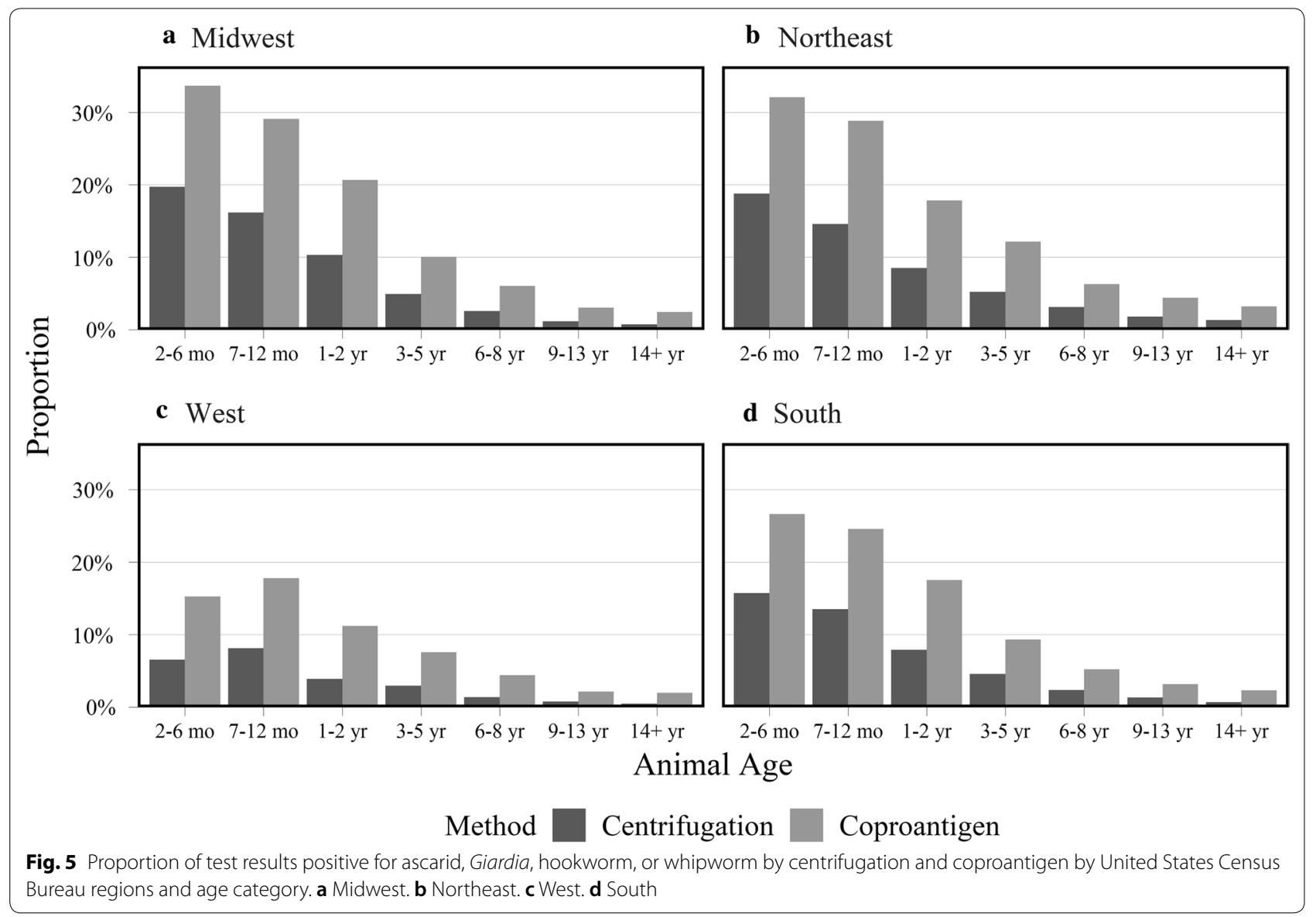

infections was in samples from 7-12-month-old cats, causing this spike in test positivity in the West.

Coproantigen-only positive results were observed more frequently than positive results for centrifugation-only. Regardless, the proportion of samples from cats that tested positive was observed to decrease with age in both coproantigen and centrifugation across all regions and parasites. This similarity in trends of test-positivity suggests that both test methods are representative of the underlying prevalence in these populations. While these data report higher levels of test positivity for coproantigen testing than centrifugation, the true disease state is 


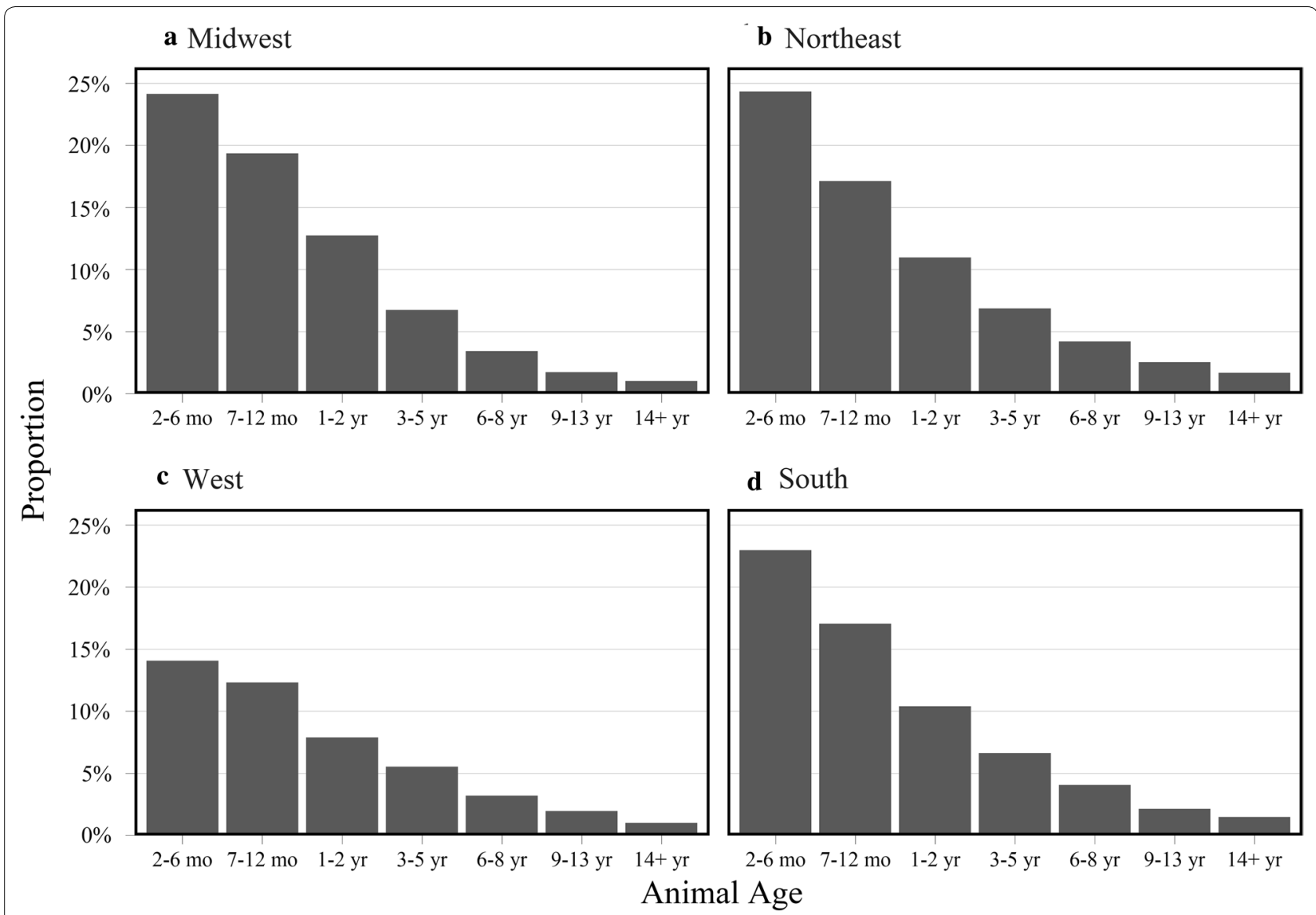

Fig. 6 Proportion of test results positive for ascarid, Giardia, hookworm, or whipworm by either centrifugation or coproantigen by United States Census Bureau regions and age category. a Midwest. b Northeast. c West. d South

not known and whether these results represent a true infection, cannot be determined from these data. It should be noted that these comparisons were between coproantigen testing and fecal flotation with centrifugation as opposed to the more commonly used in-clinic flotation, the methods and reader skill level of which may be highly variable $[15,33]$.

Previous studies have concluded that positive coproantigen results have a high level of specificity. One study tested 100 specific-pathogen-free canine fecal samples using the antigen tests for hookworm, ascarid and whipworm, and found a high specificity with all 100 samples testing negative for all three parasites [12, 34]. Additionally, Adolph et al. [14] reported the utilization of antigen detection assays for hookworm, ascarid and whipworm in conjunction with centrifugation increased the sensitivity for the detection of intestinal parasites when using necropsy as the gold standard in a cohort of 97 dogs.
There are several potential limitations to the present study. Comparisons between wellness and non-wellness positivity cannot be construed as a well versus sick comparison as the non-wellness category of visits includes procedures such as dental cleanings or surgeries in addition to clinically ill cats. The inclusion of these cases in the non-wellness category may have decreased overall positivity for this population. Although this study encompassed a large geographical population, some areas were underrepresented. Montana $(n=0)$, Mississippi $(n=14)$, North Dakota $(n=18)$, Idaho $(n=36)$, South Dakota ( $n$ $=70)$, Wyoming $(n=74)$, Arkansas $(n=87)$, Nevada $(n$ $=93)$, Kentucky $(n=97)$, Nebraska $(n=147)$, Alabama $(n=164)$ and Kansas $(n=180)$ all had fewer than 200 samples meeting inclusion criteria in 2017 and 2018. In addition, the study population consisted of data from a single commercial reference laboratory provider and thus may not reflect the true prevalence of the population. 
Table 3 Feline intestinal parasites by centrifugation or coproantigen on wellness or non-wellness veterinary visits

\begin{tabular}{|c|c|c|c|}
\hline Parasite & Classification & $\begin{array}{l}\text { Centrifugation (\%) } \\
(95 \% \mathrm{Cl})\end{array}$ & $\begin{array}{l}\text { Coproantigen (\%) } \\
(95 \% \mathrm{Cl})\end{array}$ \\
\hline \multirow[t]{2}{*}{ Ascarid } & $\begin{array}{l}\text { Other clinical } \\
\text { visit }\end{array}$ & $3.9(3.7-4.1)$ & $5.2(5.0-5.4)$ \\
\hline & Wellness & $5.3(5.1-5.5)$ & $7.2(7.0-7.4)$ \\
\hline \multirow[t]{2}{*}{ Cystoisospora } & $\begin{array}{l}\text { Other clinical } \\
\text { visit }\end{array}$ & $1.7(1.6-1.9)$ & - \\
\hline & Wellness & $2.2(2.1-2.3)$ & - \\
\hline \multirow[t]{2}{*}{ Giardia } & $\begin{array}{l}\text { Other clinical } \\
\text { visit }\end{array}$ & $1.8(1.7-2.0)$ & $6.9(6.7-7.1)$ \\
\hline & Wellness & $1.5(1.4-1.6)$ & $6.2(6.0-6.4)$ \\
\hline \multirow[t]{2}{*}{ Tapeworm } & $\begin{array}{l}\text { Other clinical } \\
\text { visit }\end{array}$ & $1.1(1.0-1.2)$ & - \\
\hline & Wellness & $0.8(0.7-0.9)$ & - \\
\hline \multirow[t]{2}{*}{ Hookworm } & $\begin{array}{l}\text { Other clinical } \\
\text { visit }\end{array}$ & $0.6(0.5-0.6)$ & $1.0(0.9-1.1)$ \\
\hline & Wellness & $0.4(0.4-0.5)$ & $0.9(0.8-1.0)$ \\
\hline \multirow[t]{2}{*}{ Lungworm } & $\begin{array}{l}\text { Other clinical } \\
\text { visit }\end{array}$ & $0.3(0.3-0.4)$ & - \\
\hline & Wellness & $0.2(0.2-0.3)$ & - \\
\hline \multirow[t]{2}{*}{ Whipworm } & $\begin{array}{l}\text { Other clinical } \\
\text { visit }\end{array}$ & $<0.1(<0.1-0.1)$ & $0.2(0.2-0.2)$ \\
\hline & Wellness & $<0.1(<0.1-0.1)$ & $0.1(0.1-0.2)$ \\
\hline
\end{tabular}

Patient history, physical examination findings, or other diagnostic tests were not available for inclusion in the analysis.

\section{Conclusions}

Intestinal parasitism in companion animals is widely considered more common in juveniles than adults [9, 35]. This study supports the previous findings that younger cats have more intestinal parasites and demonstrates specific trends in intestinal parasitism of domestic cats. Regional and age differences in parasitism help to target at-risk groups that should receive increased fecal testing. Commonly used methods of parasite detection in the USA may underestimate the risk for intestinal parasite infection in cats $[1,9]$. This study found that the percentage of samples that tested positive using coproantigen immunoassay testing was consistently higher than the percentage of samples that tested positive using flotation by centrifugation. This trend was consistent across ages, visit types and geographical regions. As a result, adding coproantigen testing to currently recommended centrifugation may lead to increased detection of parasitism in cats.

\section{Supplementary information}

Supplementary information accompanies this paper at https://doi. org/10.1186/s13071-020-04319-4.

Additional file 1: Table S1. Co-infections for hookworms, ascarids, whipworms and Giardia in feline centrifugation or coproantigen tests.

Additional file 2: Table S2. Ages of cats receiving centrifugation or coproantigen tests on wellness or non-wellness veterinary visits.

Additional file 3: Figure S1. Proportion with positive test results for centrifugal flotation and coproantigen by region broken down by United States Census Bureau region, parasite and age category.

Additional file 4: Table S3. Positive feline fecal parasite test results by centrifugation or coproantigen.

\section{Abbreviation}

CAPC: Companion Animal Parasite Council.

\section{Acknowledgements}

Not applicable.

\section{Authors' contributions}

SS, DS and EH wrote the original draft and reviewed and edited the manuscript. DS, DM and EH preformed the analysis. DK acquired data from the database. MC, DM and DK reviewed and edited the manuscript. All authors read and approved the final manuscript.

\section{Funding}

The work presented in this study was funded by IDEXX Laboratories, Inc., Westbrook, ME, USA (https://www.idexx.com/en/about-idexx/).

\section{Availability of data and materials}

All data generated or analyzed during this study are included in this published article and its additional files.

\section{Ethics approval and consent to participate}

All fecal samples were obtained from each case and submitted to a commercial reference laboratory by a practicing veterinarian during the normal diagnostic workup and monitoring of the patients in his or her care. All samples were obtained with the consent of the pet owner. To ensure privacy, additional demographic information on the pet owner, or veterinarian who submitted the sample were not collected.

\section{Consent for publication}

Not applicable.

\section{Competing interests}

The authors have an affiliation to the commercial funders of this research, as employees of IDEXX Laboratories, Inc.

Received: 20 May 2020 Accepted: 26 August 2020

Published online: 15 September 2020

\section{References}

1. Lucio-Forster A, Mizhquiri-Barbecho JS, Mohammed HO, Kornreich BG, Bowman DD. Comparison of the prevalence of Toxocara egg shedding by pet cats and dogs in the U.S.A., 2011-2014. Vet Parasitol Reg Stud Rep. 2016;5:1-13.

2. Nagamori Y, Payton ME, Duncan-Decocq R, Johnson EM. Fecal survey of parasites in free-roaming cats in northcentral Oklahoma, United States. Vet Parasitol Reg Stud Reports. 2018;14:50-3.

3. Taetzsch SJ, Bertke AS, Gruszynski KR. Zoonotic disease transmission associated with feral cats in a metropolitan area: a geospatial analysis. Zoonoses Public Health. 2018;65:412-9. 
4. Lee ACY, Schantz PM, Kazacos KR, Montgomery SP, Bowman DD. Epidemiologic and zoonotic aspects of ascarid infections in dogs and cats. Trends Parasitol. 2010;26:155-61.

5. Anderson TC, Foster GW, Forrester DJ. Hookworms of feral cats in Florida. Vet Parasitol. 2003;115:19-24.

6. Nolan TJ, Smith G. Time series analysis of the prevalence of endoparasitic infections in cats and dogs presented to a veterinary teaching hospital. Vet Parasitol. 1995;59:87-96.

7. Lucio-Forster A, Bowman DD. Prevalence of fecal-borne parasites detected by centrifugal flotation in feline samples from two shelters in upstate New York. J Feline Med Surg. 2011;13:300-3.

8. Kirkpatrick CE. Epizootiology of endoparasitic infections in pet dogs and cats presented to a veterinary teaching hospital. Vet Parasitol. 1988;30:113-24

9. De Santis AC, Raghavan M, Caldanaro RJ, Glickman NW, Moore GE. Estimated prevalence of nematode parasitism among pet cats in the United States. J Am Vet Med Assoc. 2006;228:885-92.

10. Hill SL, Cheney JM, Taton-Allen GF, Reif JS, Bruns C, Lappin MR. Prevalence of enteric zoonotic organisms in cats. J Am Vet Med Assoc. 2000;216:687-92.

11. Lightner L, Christensen BM, Beran GW. Epidemiologic findings on canine and feline intestinal nematode infections from records of the lowa state University Veterinary Clinic. J Am Vet Med Assoc. 1978;172:564-7.

12. Geng J, Elsemore DA, Oudin N, Ketzis JK. Diagnosis of feline whipworm infection using a coproantigen ELISA and the prevalence in feral cats in southern Florida. Vet Parasitol Reg Stud Rep. 2018;14:181-6.

13. Parasite Prevalence Maps. Companion Animal Parasite Council. 2019. https://capcvet.org/maps. Accessed 14 Oct 2019.

14. Adolph C, Barnett S, Beall M, Drake J, Elsemore D, Thomas J, et al. Diagnostic strategies to reveal covert infections with intestinal helminths in dogs. Vet Parasitol. 2017;247:108-12.

15. Dryden MW, Payne PA, Ridley RK, Smith VE. Gastrointestinal parasites: the practice guide to accurate diagnosis and treatment. In: Compendium on continuing education for the practicing veterinarian. Lawrenceville $\mathrm{NJ}$ : Veterinary Learning Systems; 2006.

16. Little SE, Barrett AW, Beall MJ, Bowman DD, Dangoudoubiyam S, Elsemore $D A$, et al. Coproantigen detection augments diagnosis of common nematode infections in dogs. Top Companion Anim Med. 2019;35:42-6.

17. Elsemore DA, Geng J, Cote J, Hanna R, Lucio-Forster A, Bowman DD. Enzyme-linked immunosorbent assays for coproantigen detection of Ancylostoma caninum and Toxocara canis in dogs and Toxocara cati in cats. J Vet Diagn Invest. 2017;29:645-53.

18. Elsemore DA, Geng J, Flynn L, Cruthers L, Lucio-Forster A, Bowman DD. Enzyme-linked immunosorbent assay for coproantigen detection of Trichuris vulpis in dogs. J Vet Diagn Invest. 2014;26:404-11.

19. Rishniw M, Liotta J, Bellosa M, Bowman D, Simpson KW. Comparison of 4 Giardia diagnostic tests in diagnosis of naturally acquired canine chronic subclinical giardiasis. J Vet Intern Med. 2010;24:293-7.

20. R Development Core Team. R: A language and environment for statistical computing. Vienna: R Foundation for Statistical Computing; 2014. http:// www.R-project.org/.
21. Dowle M, Srinivasan A, Gorecki J, Chirico M, Stetsenko P, Short T, et al. data.table: extension of "data.frame." 2019. v1.12.16 https://cran.r-proje ct.org/web/packages/data.table/index.html. Accessed 13 Sept 2019.

22. Bache SM, Wickham H. magrittr: a forward-pipe operator for R. 2014. v1.5. https://cran.r-project.org/web/packages/magrittr/index.html. Accessed 13 Sept 2019

23. Müller K. here: a simpler way to find your files. 2017. v0.1. https://cran.rproject.org/web/packages/here/index.html. Accessed 13 Sept 2019.

24. Harrell FE. Hmisc: Harrell miscellaneous. 2019. v4.2. https://cran.r-proje ct.org/web/packages/Hmisc/index.html. Accessed 13 Sept 2019.

25. Wickham H, Chang W, Henry L, Pedersen TL, Takahashi K, Wilke C, et al. ggplot2: create elegant data visualisations using the grammar of graphics. 2019. v3.2.1. https://cran.r-project.org/web/packages/ggplot2/index .html. Accessed 13 Sept 2019.

26. Auguie B, Antonov A. gridExtra: miscellaneous functions for "grid" graphics. 2017. v2.3. https://cran.r-project.org/web/packages/gridExtra/index .html. Accessed 13 Sept 2019.

27. Kassambara A. ggpubr: "ggplot2" based publication ready plots. 2019. v0.2.3. https://cran.r-project.org/web/packages/ggpubr/index.html. Accessed 13 Sept 2019.

28. Chang W. extrafont: tools for using fonts. 2014. v0.17. https://cran.r-proje ct.org/web/packages/extrafont/index.html. Accessed 13 Sept 2019.

29. United States Census Bureau 2019. https://www.census.gov/en.html. Accessed 9 July 2019

30. McNamara J, Drake J, Wiseman S, Wright I. Survey of European pet owners quantifying endoparasitic infection risk and implications for deworming recommendations. Parasit Vectors. 2018;1 1:571.

31. Bowman DD. Georgis' parasitology for veterinarians. 10th ed. St. Louis, MO: Elsevier; 2019.

32. Dryden MW, Payne PA, Smith V. Accurate diagnosis of Giardia spp. and proper fecal examination procedures. Vet Ther. 2006;7:4-14.

33. Dryden MW, Payne PA, Ridley R, Smith V. Comparison of common fecal flotation techniques for the recovery of parasite eggs and oocysts. Vet Ther. 2005;6:15-28

34. Geng J, Hanna R, Elsemore D. Demonstration of the specificity of the Fecal Dx (hookworm, ascarid, and whipworm antigen) assays. 2017. American Association of Veterinary Parasitologists, 62nd Annual Meeting. Indianapolis, IN USA. https://www.aavp.org/documents/2017/08/aavp2017-annual-meeting-proceedings.pdf/.

35. Gates MC, Nolan TJ. Endoparasite prevalence and recurrence across different age groups of dogs and cats. Vet Parasitol. 2009;166:153-8.

\section{Publisher's Note}

Springer Nature remains neutral with regard to jurisdictional claims in published maps and institutional affiliations.

\footnotetext{
Ready to submit your research? Choose BMC and benefit from:

- fast, convenient online submission

- thorough peer review by experienced researchers in your field

- rapid publication on acceptance

- support for research data, including large and complex data types

- gold Open Access which fosters wider collaboration and increased citations

- maximum visibility for your research: over 100M website views per year
}

At BMC, research is always in progress.

Learn more biomedcentral.com/submissions 